\title{
Marcapasso com Sensor de Contratilidade Regulado pelas Variações do Sistema Nervoso Autônomo na Miocardiopatia Chagásica Crônica
}

\author{
Oswaldo Tadeu Greco, Roberto Vito Ardito, Dorotéia Rossi Silva Souza, Max Schaldach
}

São José do Rio Preto, SP

\begin{abstract}
Objetivo - Analisar o desempenho da estimulação cardíaca artificial com marcapasso do tipo VVIR cujo sensor é regulado pelas variações do sistema nervoso autônomo em pacientes chagásicos com distúrbio no sistema de condução.

Métodos - Estudados 47 chagásicos, 28 do sexo masculino, com idades entre 24 e 68 anos, 36 tinham bloqueio atrioventricular (AV) total; 8, bloqueio AV de $2^{\circ}$ grau 2; e 3 doença do nódulo sinusal, e encontravam-se, de acordo com a NYHA, em classe I (4), II (15), III (16) eIV (12). Após o implante de marcapasso do tipo VVIR os pacientes foram acompanhados durante 12 meses. A resposta de freqüencia foi registrada em gravações de Holter de 24 h e divididos em dois grupos de acordo com a FC em repouso - grupo 1: >65bpm e grupo 2: $\leq 65$ bpm, para estudo comparativo, considerando: 1) FC em exercício no periodo de pósimplante; 2) PA em repouso após o implante e 3) avaliação dos grupos de eletrodos identificados como TIR-60UP e outros eletrodos.

Resultados-O grupo 1 teve em exercício menor variação entre seus valores, do que o grupo 2, indicando que esse tipo de sistema de estimulação permite controlar individualmente cada paciente. Os valores de PA em repouso e em exercício não foram diferentes entre os grupos. $O$ eletrodo do tipo TIR-60-UP, comportou-se como os demais eletrodos.

Conclusão- O marcapasso do tipo VVIR cujo sensor é regulado pelas variações do SNA propicia o restabelecimento dos mecanismos fisiológicos em chagásicos, sendo que $74 \%$ deles tiveram melhora de uma ou duas classes funcionais da NYHA.
\end{abstract}

Palavras-chave: marcapasso, doença de Chagas, marcapasso com resposta de frequiência, sistema nervoso autônomo

\section{Chagas Heart Disease and Contractility Rate Responsive Pacing Controlled by Autonomic Nervous System Variations}

\begin{abstract}
Purpose-To analyse the performance of the artificial cardiac stimulation with the VVIR pacemaker whose sensor is adjusted by the variations of the autonomic nervous system in Chagas disease patients with deficiency of the conduction system.

Methods - Forty-seven Chagas disease patients have been studied, 28 male between 24 and 68 years old, 36 patients had complete AV block, 8 had $2^{\text {nd }}$ degree AV block and the other 3 had sinus node disease. The patients were in class I (4), II (15), III (16) and IV (12) according to the NYHA. A 12-month-follow-up with constant clinical evaluations was carried out after pacemaker implantation. Patients were divided in 2 different groups according to the HR at rest - group 1: $>65$ beats per minute (bpm) and group 2: $\leq 65 \mathrm{bpm}$, for a comparative study considering: 1) HR at stress test after the implantation; 2) arterial blood pressure at rest after the implantation and, 3) evaluation of the identified electrodes such as TIR-60-UP and others.

Results - The group 1 had greater HR at rest, and a smaller variation of values at stress than group 2 . This shows that with this type of stimulation system it is possible to control each patient separately. The values of blood pressure at rest and during stress were not different between groups. According to the factors analysed the TIR-60-UP electrode had the same performance as the others.

Conclusion - The VVIR pacemaker with the sensor adjusted by the ANS variations has provided the Chagas patients with a restoration of their physiological mechanisms. 74\% of them had the improvement of either one or 2 functional classes.
\end{abstract}

Key-words: pacing, Chagas' disease, rate responsive pacing, autonomic nervous system

Arq Bras Cardiol, volume 71 (n 6), 751-761, 1998

Instituto de Moléstias Cardiovasculares - São José do Rio Preto

Correspondência: Oswaldo Tadeu Greco - Instituto de Moléstias Cardiovasculares - Caixa Postal, 681 - 15015-210 - São José do Rio Preto, SP

Recebido para publicação em 3/11/98

Aceito em 14/10/98
O primeiro marcapasso cardíaco artificial foi desenvolvido em 1958 por Elmqvist e implantado por Senning, tornando-se, a partir daí, a terapia de escolha para as bradiarritmias. Com o advento do transistor foi possível a fabricação 


\begin{tabular}{|c|c|c|c|c|}
\hline \multicolumn{5}{|c|}{ Quadro I } \\
\hline $1^{\mathrm{a}}$ Letra & $2^{\mathrm{a}}$ Letra & $3^{a}$ Letra & $4^{\mathrm{a}}$ Letra & $5^{\mathrm{a}}$ Letra \\
\hline $\begin{array}{l}\text { Câmara } \\
\text { estimulada }\end{array}$ & $\begin{array}{l}\text { Câmara } \\
\text { detectada ou } \\
\text { monitorada }\end{array}$ & $\begin{array}{l}\text { Modo de } \\
\text { resposta do } \\
\text { marcapasso }\end{array}$ & $\begin{array}{l}\text { Parâmetros } \\
\text { programáveis }\end{array}$ & $\begin{array}{l}\text { Funções } \\
\text { especiais para } \\
\text { taquiarritmia }\end{array}$ \\
\hline Segunda letra: & $\begin{array}{l}\mathrm{A} \text { - átrio } \\
\mathrm{V} \text { - ventrículo } \\
\mathrm{D} \text { - ambas as c } \\
\mathrm{T} \text { - sincronizac } \\
\mathrm{O} \text { - nenhuma }\end{array}$ & $\begin{array}{l}\text { culo } \\
\text { o deflagra seu }\end{array}$ & idade elétrica é & \\
\hline Terceira letra: & \multicolumn{4}{|c|}{$\begin{array}{l}\text { I - inibido: o gerador de pulso fica inibido frente a uma atividade espontânea detectada. } \\
\text { D - dupla resposta: quando pode ser inibido e sincronizado } \\
\text { O - não se aplica } \\
\text { T - sincronizado: o gerador de pulso deflagra seu pulso quando uma atividade elétrica é detectada. }\end{array}$} \\
\hline Quarta letra: & \multicolumn{4}{|c|}{$\begin{array}{l}\text { O - não há parâmetro programável } \\
\mathrm{P} \text { - gerador de pulso programável em até } 2 \text { parâmetros } \\
\mathrm{M} \text { - gerador de pulso programável em } 3 \text { ou mais parâmetros } \\
\mathrm{R} \text { - com modulação de frequiência }\end{array}$} \\
\hline Quinta letra: & $\begin{array}{l}\mathrm{O} \text { - nenhuma } \\
\mathrm{B} \text { - trem de pu } \\
\mathrm{N} \text { - competiçã } \\
\mathrm{S} \text { - resposta en } \\
\mathrm{E} \text { - controle ex }\end{array}$ & $\begin{array}{l}\text { (burst) } \\
\text { do paciente ( } \\
\text { com pulsos } \\
\text { erador de puls }\end{array}$ & $\begin{array}{l}\text { lemanda") } \\
\text { mento cada vez }\end{array}$ & \\
\hline
\end{tabular}

de marcapassos implantáveis, pois os circuitos usados até então, denominados VOO (estimulação ventricular com freqüência fixa), eram simples e serviam apenas para controlar a frequiência de estimulação ventricular (quadro I). Entretanto, muitos pacientes apresentavam ritmos espontâneos, tornando, nesse caso, freqüente a competição entre o ritmo natural do coração e o marcapasso. Fato esse solucionado na década de 60 com o desenvolvimento de dois outros modos de estimulação, o ventricular deflagrado (VVT - estimulação ventricular deflagrada) e o ventricular inibido(VVI - estimulação ventricular inibida pelo QRS), com amplificador de eletrocardiograma e circuito para detectar o QRS ${ }^{1}$.

No início dos anos 70, com a incorporação dos circuitos integrados, começaram a surgir os marcapassos multiprogramáveis, assim denominados pela propriedade de programação externa de vários parâmetros. A adição dessa programação tornou mais fácil o acompanhamento dos pacientes, por meio de freqüentes ajustes dos parâmetros. Na década de 80 , a introdução da bateria de lítio possibilitou aumento da longevidade desses geradores, já em destaque por sua multiprogramabilidade, como demonstrado por Pless e $\mathrm{col}^{2}$.

O próximo passo foi o desenvolvimento da estimulação de dupla câmara, oferecendo estimulação e inibição combinadas para átrio e ventrículo (DDD - estimulação bicameral inibida por estímulos espontâneos). Este tipo de estimulador evoluiu muito, tanto nas características dos geradores, como na expansão de indicações, facilitando o acompanhamento dos pacientes no pós-operatório. Além da sofisticação tecnológica dos marcapassos, esses apare- lhos são hoje bem menores que os iniciais, mais confiáveis e oferecem qualidade de estimulação mais satisfatória com diferentes modos de estimulação ${ }^{3}$.

Alguns autores têm demonstrado melhor desempenho hemodinâmico oferecido pelos marcapassos modernos, mais fisiológicos, outros defendem a utilização de marcapassos ventriculares mais acessíveis, de simples manuseio e implantação, obtendo resultados hemodinâmicos muito semelhantes com ambos os tipos ${ }^{4}$.

A evolução da estimulação cardíaca artificial, associada a estudos multidisciplinares, principalmente com o progresso da microeletrônica, oferece sistemas de qualidade que permitem maior segurança no acompanhamento dos pacientes. O desenvolvimento tecnológico, tanto dos geradores de pulso, como dos eletrodos, criou condições eletroquímicas e eletrofisiológicas para o contato ideal entre sua superfície e o miocárdio ${ }^{5}$.

É reconhecido que a seqüência de contração no coração é controlada por mecanismos autônomos, que estão sujeitos a influências reguladoras extracardíacas. Por meio da inervação simpática, estes mecanismos permitem adaptação inotrópica e cronotrópica do débito cardíaco (DC) para o trabalho físico e variações neuro-humorais. Estes mecanismos de compensação são eficazes perante um miocárdio sadio, que durante o exercício físico sofre aumento da freqüência cardíaca (FC), que contribui na adaptação do DC, restabelecendo a excitação fisiológica, o que é evidenciado pela estimulação cardíaca artificial associada a um sensor ligado a parâmetros metabólicos, hemodinâmicos e cardiovasculares ${ }^{6}$. 
Este sistema, de fundamental importância na regulação de vários órgãos e sistemas e, principalmente, nas funções que devem ser controladas continuamente, é representado pelos sistemas simpático e parassimpático, controlando as variações do sistema cardiovascular. Em relação a este sistema, a regulação da pressão arterial (PA) é feita por interação entre cronotropismo, inotropismo e resistência vascular periférica, sendo o SNA o principal agente desse processo. Desta forma, é natural o crescente interesse pela avaliação funcional do SNA, tanto para o conhecimento da fisiologia cardíaca, como para sua aplicação clínica.

O coração é inervado por uma rede complexa de nervos por meio de vias discretas, mas sobrepostas, que variam substancialmente entre as espécies. As fibras eferentes vagais (SNA parassimpático) e simpáticas (SNA simpático) transmitem impulsos nervosos para o coração e modulam as propriedades excitáveis cardíacas, enquanto as fibras nervosas aferentes transmitem impulsos a partir do coração e são responsáveis pelos reflexos cardíacos. O nódulo sinusal, estrutura formadora do impulso cardíaco, é ricamente enervado por terminações nervosas colinérgicas e adrenérgicas pós-ganglionares, algumas das quais em contato direto entre elas ${ }^{7}$.

Em geral, o tônus vagal é antiarrítmico, em virtude de ações eletrofisiológicas diretas no coração e ações indiretas por antagonizar os efeitos simpáticos, pode facilitar o aparecimento de arritmia ventricular, provocada pela bradicardia. Sugere-se que a estimulação simpática também pode ser antiarrítmica por melhorar a contratilidade e o fluxo coronário no coração em falência, mas a demanda miocárdica de oxigênio aumentada pode causar algumas arritmias cardíacas ${ }^{8}$.

A morte súbita no curso da doença de Chagas e do infarto agudo do miocárdio (IAM), também é freqüentemente associada a disfunção autonômica ${ }^{9}$. A isquemia e o IAM podem provocar também instabilidade elétrica das fibras autonômicas intramiocárdicas, favorecendo o aparecimento de arritmias cardíacas ${ }^{9}$.

Lesões do sistema nervoso autonômico e especialmente intracardíaco são ocorrências constantes na miocardiopatia chagásica, tanto em sua fase aguda, como em sua evolução para cronificação. Estes achados são mais freqüentes em pacientes que apresentam insuficiência cardíaca (IC), um grupo representativo nesta doença. Entretanto, também são encontrados em chagásicos com distúrbios de condução do ritmo, com ou sem IC, aparentemente assintomáticos, mas candidatos a morte súbita.

O mecanismo patogênico das alterações funcionais e morfológicas encontradas na doença de Chagas é um assunto complexo e controverso. As alterações mais freqüentes são as inflamatórias e as nervosas, reconhecidas na fisiopatologia da forma crônica de tripanossomíase americana. Para Mott e Hagstrone as alterações morfológicas tardias dependem do parasitismo que envolve as fibras miocárdicas e das lesões de sensibilização na fase aguda da doença, caracterizando-a como uma miocardite intersticial crônica difusa. No que se refere a extensão e a gravidade das lesões, admite-se que a miocardite aguda chagásica pode ser explicada pela sensibilização causada por proteínas heterólogas liberadas no nível das células destruídas pelo parasitismo, provocando lesões isquêmicas e fenômenos de infiltração. Constitui também fase importante nos processos patogênicos da doença de Chagas a liberação de proteínas estranhas resultantes de Leishmanias que se abrigam em células destruídas ${ }^{11}$.

Baseados em estudos quantitativos sobre a denervação parassimpática, Amorin e col estabeleceram uma cronologia de eventos na evolução da cardiopatia chagásica, definidos em três fases: a) neurogênica pura, com transtornos de ritmo; b) vascular, com insuficiência coronária relativa, e c) miogênica, com lesões pós-isquêmicas acentuadas. Consideram que na miocardiopatia chagásica crônica o distúrbio autonômico e/ou a miocardite fibrosante crônica e a disfunção ventricular poderiam atuar como fatores predisponentes a maior risco de morte súbita, privando seus portadores de vida normal ${ }^{12}$.

Alterações como encarquilhamento, tumefação e vacuolização do pericárdio e picnose também foram detectadas por Amorin e col, tanto em gânglios de casos normais como em chagásicos, embora mais freqüentemente nestes últimos ${ }^{13}$. Estas alterações parecem inespecíficas e são possivelmente relacionadas com a hipóxia e outras agressões que podem ocorrer nos períodos finais da vida, além de prováveis sobreposições de fenômenos ulteriores à morte. Esse tipo de denervação, em evolução na fase crônica da doença sem relação com o processo inflamatório, poderia ser conseqüente a alterações de estruturas nervosas ganglionares. Assim, esses autores sugerem que no chagásico crônico a despovoação neuronal do núcleo dorsal do vago poderia ser responsável por parte das alterações do sistema nervoso autônomo intracardíaco ${ }^{14}$.

\section{Métodos}

A partir de agosto/91 iniciou-se no Instituto de Moléstias Cardiovasculares de São José do Rio Preto (IMC) o implante de marcapasso do tipo VVIR em pacientes chagásicos, com um tipo de sensor de contratilidade(*). Este estudo mostra a evolução dos primeiros 47 pacientes, sendo $28(60 \%)$ do sexo masculino e 19 (40\%) do feminino, com idades entre 24 e 68 (médias de 49,9 e 45,7 anos, respectivamente), sendo que $18(38 \%)$ tinham implantes prévios, enquanto $29(62 \%)$ foram submetidos ao primeiro implante. Foram utilizados eletrodos do tipo TIR-60-UP em 26 (55\%) pacientes, e os 21 (45\%) restantes receberam eletrodos de outros tipos, como:DNP.60.A, Medtronic 6971 e 5026, PS.60.PeQT611.

O implante foi indicado após exame clínico e ecocardiografia bidimensional, revelando que 35 (73\%) apresentaram área cardíaca normal e $12(27 \%)$ alterada, com índices cardiotorácicos, variando de 0,35 a 0,62. A fração de ejeção mostrou-se alterada em $30(64 \%)$ pacientes, com variação de 40 a $55 \%$, com média de $49 \%$ e normal em 17 (36\%), variando de 56 a $62 \%$, com média de $58 \%$. O implante do marcapasso

(*) Biotronik, Neos - PEP 
foi indicado por bloqueio atrioventricular (AV) em 44 (94\%) dos casos, sendo que $36(77 \%)$ tinham bloqueio AV total (grau 3), oito (17\%) bloqueio AV de $2^{\circ}$ grau e três $(6 \%)$ apresentaram doença do nódulo sinusal. Em semelhança à classificação clínica funcional da New York Heart Association (NYHA), quatro (8\%) estavam na CF I, 15 (32\%) na II, 16 (34\%) naIII $12(26 \%)$ naIV.

O protocolo de acompanhamento foi iniciado após alta hospitalar, permanecendo o paciente em modo VVI de estimulação (estimulação ventricular inibida pelo QRS) durante 15 dias. A partir da primeira revisão do marcapasso, começaram ser analisados e colocados em sua memória os dados obtidos do paciente durante diversos tipos de atividades diárias.

Em cada revisão, o paciente foi submetido a estudo ecocardiográfico bidimensional, Holter de $24 \mathrm{~h}$ (registrado pelo próprio gerador) e teste ergométrico em bicicleta com carga de 25 a 100W (protocolo modificado de Bruce), a fim de comparar os dados fornecidos pela variação do sistema nervoso simpático. Durante este estudo foram avaliados também FC ePA, ambas em repouso e em exercício, em até12 meses após o implante do marcapasso. Devido a comportamentos clínicos diferentes durante a estimulação cardíaca em repouso, os pacientes foram divididos em dois grupos: grupo 1, pacientes com FC >65bpm e grupo 2, pacientes com $\leq 65 \mathrm{bpm}$.

Os pacientes receberam eletrodos transvenosos unipolares implantados na ponta do ventrículo direito(VD), com a finalidade de medir a impedância dessa cavidade. O sistema consiste em uma corrente constante de $4.096 \mathrm{~Hz}$ e $20 \mathrm{~A}$, que forma uma onda quadrada de excitação muscular usada para determinar a impedância entre a ponta do eletrodo e a loja do gerador. As informações foram analisadas durante o estudoe ajustadas de acordo com as variações do ciclo cardíaco. Neste caso, os estímulos são transmitidos pelo polo cátodo, em contato com o miocárdio. A resposta da excitabilidade do miocárdio depende, principalmente, do tamanho da área que está em contato com sua superfície (fig. 1).

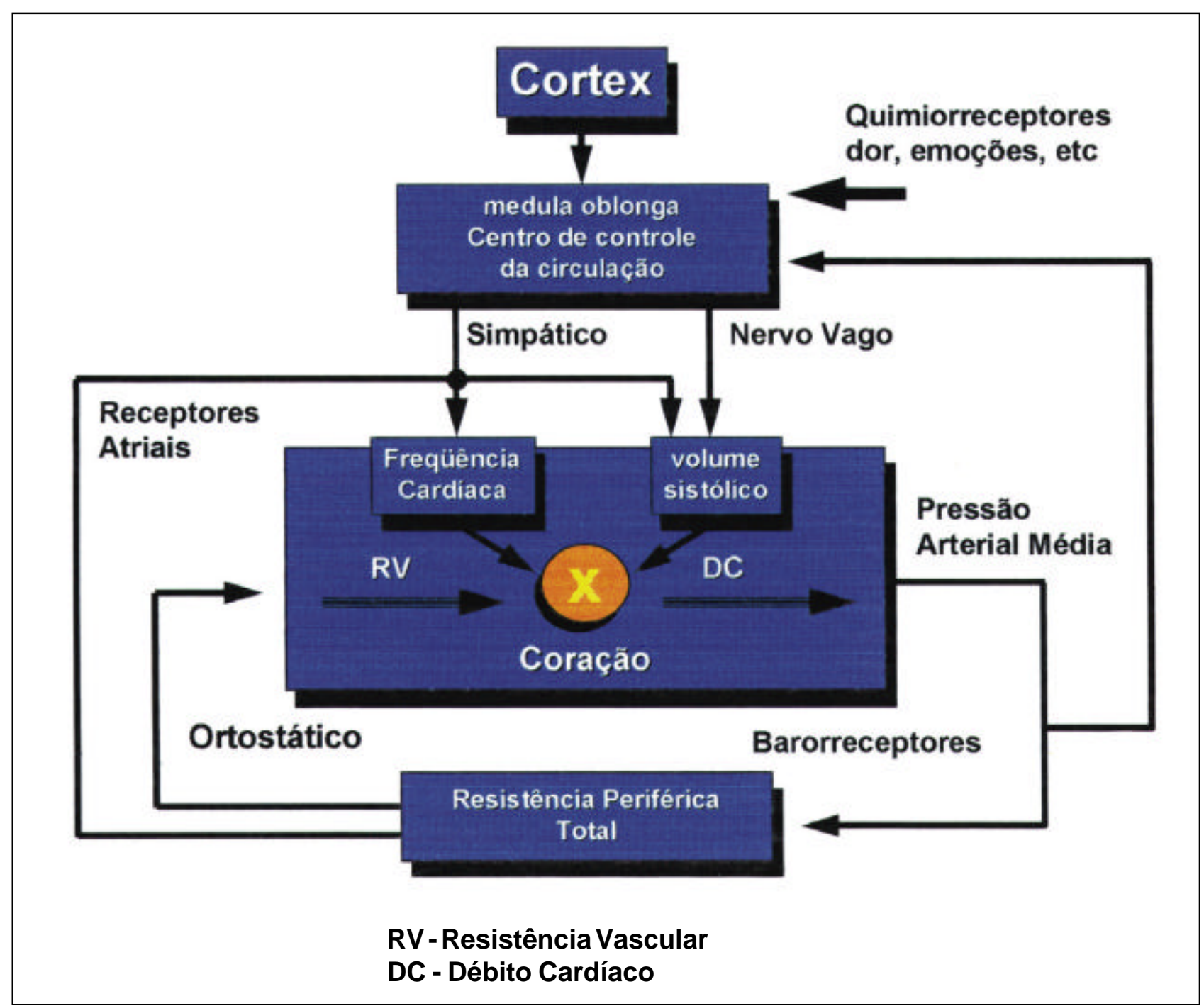

Fig. 1 - Representação esquemática do sistema de alça fechada que recebe interferências externas, levando-as ao sistema nervoso central que as transmite ao coração, cuja freqüência de estimulação é então reprogramada, modificado de Schaldach, 1993. RV-resistência vascular; DC- débito cardíaco. 
Os eletrodos utilizados neste estudo caracterizaram-se pelo revestimento com nitrato de irídio e titânio, depositados no sustrato com o auxílio de vaporização física e química. A condensação deste material é restrita ao substrato que é mantido em baixa temperatura, permitindo que essa camada cresça em forma de colunas separadas por pequenos espaços. Esse processo cria um sustrato poroso, o que facilita seu contato com o miocárdio, levando simultaneamente a perfeita estimulação e sensibilidade, a essa superfície.

O sensor Neos-PEP (Biotronik) empregado no presente estudo foi programado para manter estimulação constante por meio de período refratário de $350 \mathrm{~ms}$, largura do pulso de $0,50 \mathrm{~ms}$, amplitude de 4,8 e sensibilidade de 2,4, apresentando também: a) sistema de medida de impedância integrada a um sistema analógico de conversão digital; b) capacidade adicional de memória para arquivar os dados da impedância; c) características especiais de interrogação capazes de transmitir os dados memorizados para um programador externo, e d) circuito de processamento dos dados com sua transformação em uma curva de resposta de freqüência.

Além disso, esse tipo de biossensor, também chamado de closed-loop control, tem sua programação modificada conforme a variação dos diversos parâmetros que interferem no sistema nervoso autônomo. Assim, para avaliação e melhor ajuste da programação do biossensor à necessidade do paciente, quanto ao aumento ou diminuição da freqüência estimulada, foi considerado o maior número possível de dados obtidos do paciente em diversas atividades diárias, como repouso, exercício e outro tipo de atividade física diária.

A impedância cardíaca foi medida por injeção de uma corrente alternante de $40 \mathrm{uA}$ no eletrodo, corrente esta menor que o limiar de estimulação, mas alta o suficiente para detectar com segurança pequenas variações da impedância associadas à sístole. Após a amplificação e a filtragem, o sinal foi detectado por medida da voltagem produzida pelas mudanças da impedância. O sinal da impedância foi processado empregando um filtro especial, ampliado e ajustado em oito pontos e, em seguida, encaminhado para análise no sistema digital processador dos sinais.

Para minimizar a corrente que opera esse tipo de marcapasso, a medida da impedância é restrita na porção do ciclo cardíaco, durante o final do PEP (período de pré-ejeção). Na realidade, os processos que regulam o próprio organismo ocorrem relativamente devagar, a tal ponto que as medidas podem ser facilmente obtidas. Caso apareça algum distúrbio que dificulte a determinação do PEP, automaticamente é aumentada sua sensibilidade.

Medidas estáveis da impedância facilitam o trabalho do sistema de registro do marcapasso e determinam redução do consumo de energia. Este circuito permite armazenar sinais não filtrados, possibilitando interrogar os dados pela unidade de programação, em duas situações referidas: a) quando a função PEP é desligada o sistema torna-se semelhante a um marcapasso multiprogramável normal; b) quando o controle PEP é estimulado, é possível acionar uma série de funções, como: 1) registrar dados da impedância; 2) inter-
Marcapasso com sensor de contratilidade na doença de Chagas

rogar estes dados da memória; 3) registrar os dados obtidos na sua memória; 4) contar o número de eventos; 5) analisar as variações do PEP.

A análise exploratória dos dados consistiu em: 1) estudos comparativos pela aplicação de teste não paramétrico de Mann-Whitney considerando: a) grupo de eletrodos do tipo TIR-60-UP e grupo representado por outros eletrodos, com base na diferença entre FC em exercício e em repouso de cada paciente; b) FC em exercício no período de pós-implante, referente ao grupo 1 , representado por pacientes com $\mathrm{FC}$ em repouso $>65 \mathrm{bpm}$ e ao grupo 2, pacientes com esse parâmetro $\leq 65 \mathrm{bpm}$; c) PA em repouso no período de pós-implante, referente aos grupos 1 e 2 .

Considerou-se no estudo descritivo os valores de FC e PA em repouso e exercício no pós-implante, identificandose mediana, perfis equivalentes ao primeiro quartil (valor da amostra ordenada que deixa $25 \%$ dos valores abaixo dele) e $3^{\circ}$ quartil (valor da amostra ordenada que deixa $75 \%$ dos valores abaixo dele) e valores máximo e mínimo, com acompanhamento dos pacientes desde a data do implante até 12 meses de seguimento. Neste caso, foi utilizada a representação esquemática conhecida por box-plot, com indicação de média, mediana, dispersão dos dados, caudas e dados discrepantes. O valor de PA pós-implante empregado para os cálculos foi obtido do valor médio entre pressões sistólica e diastólica.

Com relação ao cálculo do coeficiente de correlação de Pearson, neste estudo foi analisada a dependência linear entre FC e medida de PA no repouso e em exercício, considerando-se os grupos 1 e 2.

Foi adotado nível de $5 \%$ de significância nos testes estatísticos.

\section{Resultados}

A avaliação clínica dos 47 pacientes realizada em seguimento de 12 meses, após o implante de marcapasso VVIR com eletrodos do tipo TIR-60-UP e outros tipos, considerou valores de FC e PA, ambas em repouso e exercício físico sob carga de 25 a $100 \mathrm{~W}$, além do desempenho do marcapasso, avaliado também pelas respostas de frequiências obtidas por Holter de $24 \mathrm{~h}$.

Há evidências de comportamentos distintos entre os grupos com respeito a FC em exercício ( $\mathrm{p}=0,0004)$, o que é evidenciado na tabela I, que mostra o grupo 1 com valores mais baixos de FC durante exercício físico, em comparação ao

\begin{tabular}{|c|c|c|c|c|c|c|}
\hline \multicolumn{7}{|c|}{$\begin{array}{c}\text { Tabela I - Mediana, quartis }(\mathrm{Q} 1 \mathrm{e} Q 3) \text { e valores mínimo e máximo } \\
\text { para freqüência cardíaca em batimentos por minuto }(\mathrm{bpm}) \text { durante } \\
\text { exercício, no grupo } 1 \text { e no grupo } 2, \text { com } \mathrm{p}=0,0004 \text {. } \mathrm{N} \text { - número de } \\
\text { pacientes }\end{array}$} \\
\hline $\begin{array}{c}\text { Grupo } \\
\mathrm{N}^{\circ}\end{array}$ & $\mathrm{N}$ & Mediana & $\begin{array}{c}\text { Q1 } \\
(25 \%)\end{array}$ & $\begin{array}{c}\text { Q3 } \\
(75 \%)\end{array}$ & Mínimo & Máximo \\
\hline 1 & 16 & 100 & 95 & 110 & 75 & 125 \\
\hline
\end{tabular}




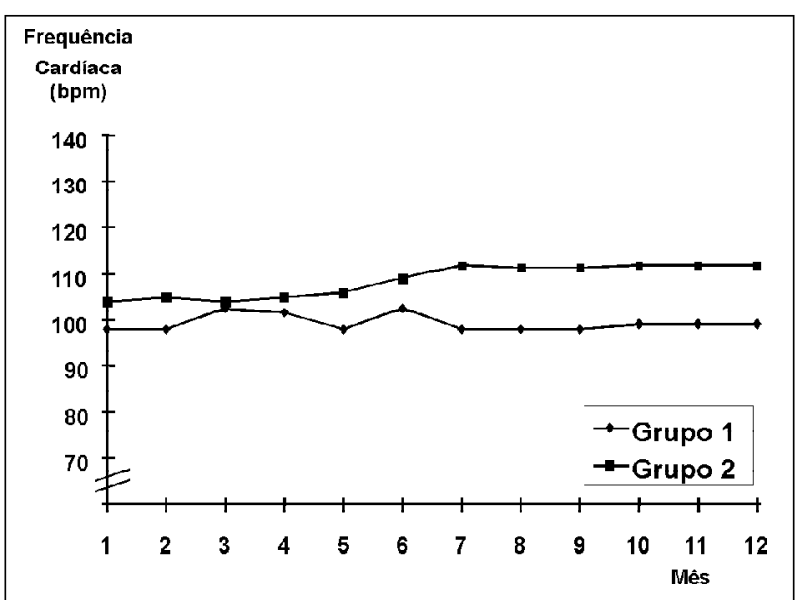

Fig. 2 - Perfil médio da frequiência cardíaca durante exercício para o grupo 1 e grupo 2 , em 12 meses de evolução.

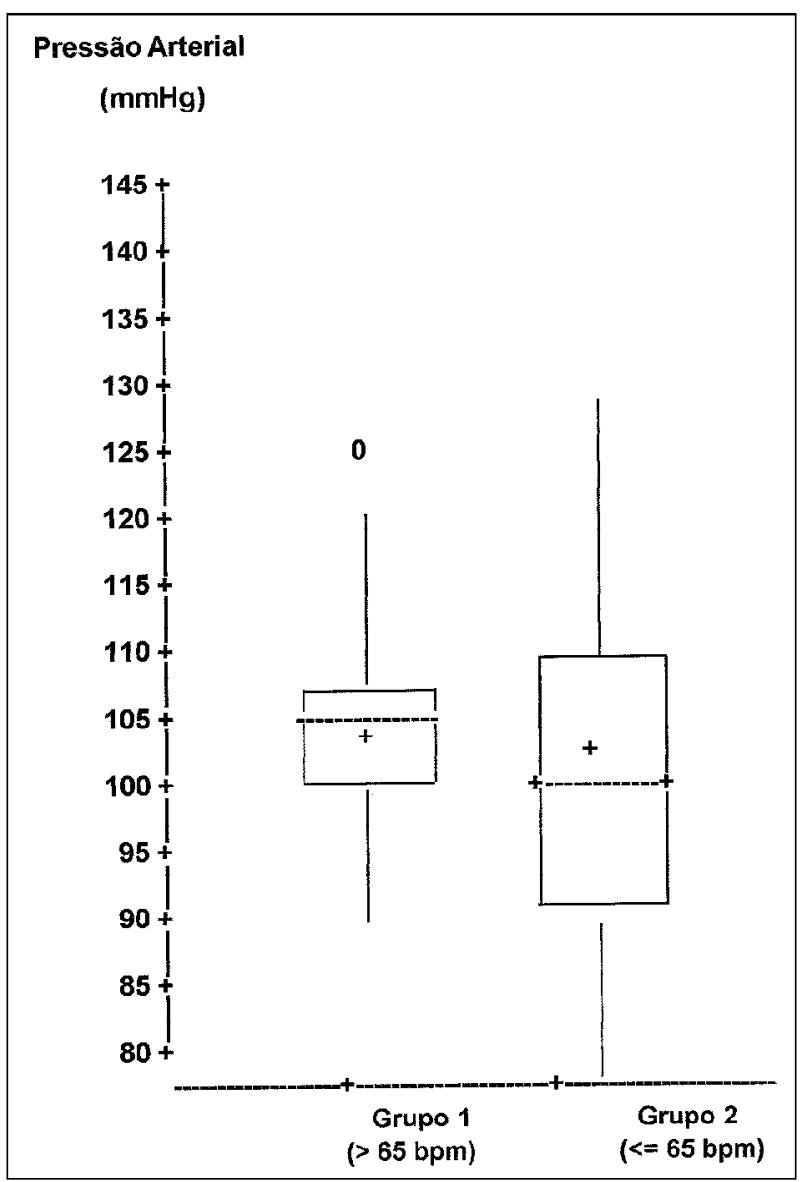

Fig. 3 - Distribuição dos valores de pressão arterial durante repouso, para o grupo 1 e grupo 2. Valor médio (+); Mediana (---).

Tabela II - Mediana, quartis (Q1e Q3) e valores mínimo e máximo de pressão arterial $(\mathrm{mmHg})$ em repouso para o grupo 1 e grupo 2 . *Nnúmero de pacientes

\begin{tabular}{|ccccccc|}
\hline Grupo & $\mathrm{N}$ & Mediana & $\begin{array}{c}\text { Q1 } \\
(25 \%)\end{array}$ & $\begin{array}{c}\text { Q3 } \\
(75 \%)\end{array}$ & Mínimo & Máximo \\
\hline 1 & 16 & 105 & 100 & 108 & 80 & 125 \\
2 & 31 & 100 & 90 & 110 & 80 & 130 \\
\hline
\end{tabular}

grupo 2. Enquanto no grupo 1,75\% (quartil 3) dos pacientes atingiram até $110 \mathrm{bpm}$, no grupo 2 esse valor foi de $120 \mathrm{bpm}$.

A figura 2 apresenta o perfil médio da FC dos pacientes em exercício, de ambos os grupos, durante os 12 meses de estudo. Nota-se maior instabilidade nos pacientes com FC em repouso >65bpm (grupo 1), em comparação àqueles com valor $\leq 65 \mathrm{bpm}$ (grupo2). Entretanto, aos sete meses de evolução ambos os grupos atingiram estabilidade, embora com leve aumento de valores no grupo 2 em relação ao início do estudo, enquanto do grupo 1 esses valores foram comparáveis ao período inicial.

Embora tenha se verificado menor FC em exercício em pacientes no grupo 1, o mesmo não ocorreu quando calculada a diferença entre os valores de FC em exercício e repouso. Istoé, os pacientes que em repouso apresentaram menor FC (grupo 2), considerando os valores em exercício apresentaram diferença significativamente maior entre os valores de FC, do que aqueles do grupo 1 (FC em repouso $>65 \mathrm{bpm})(\mathrm{p}=0,0001)$. Nota-se, portanto, que os pacientes do grupo 1, apresentaram em exercício, FC reduzida, em comparação aos do grupo 2, sugerindo que os marcapassos do grupo 1 foram mantidos com baixa resposta à estimulação, em relação aos do grupo 2.

Com relação a PA em repouso, não houve indicação de diferença entre os grupos pela aplicação do teste não paramétrico $(\mathrm{p}=0,36)$. A figura 3 e a tabela II mostram a análise descritiva e a distribuição dos valores da PA em repouso, respectivamente, em ambos os grupos. O grupo 1 destaca-se pela menor variação de valores, ou seja, os pacientes $>65 \mathrm{bpm}$ apresentaram em repouso valores de pressão mais próximos entre si, que o grupo $2 \mathrm{com}$ freqüência $\leq 65 \mathrm{bpm}$, cujo valor máximo foi mais elevado que no grupo 2 . A figura 4 apresenta o perfil médio dos grupos $1 \mathrm{e} 2$, para PA em repouso, revelando semelhança entre eles durante os $12 \mathrm{me}$ ses de estudo.

A PA em exercício também não foi fator de diferenciação entre os grupos, com probabilidade de significância de

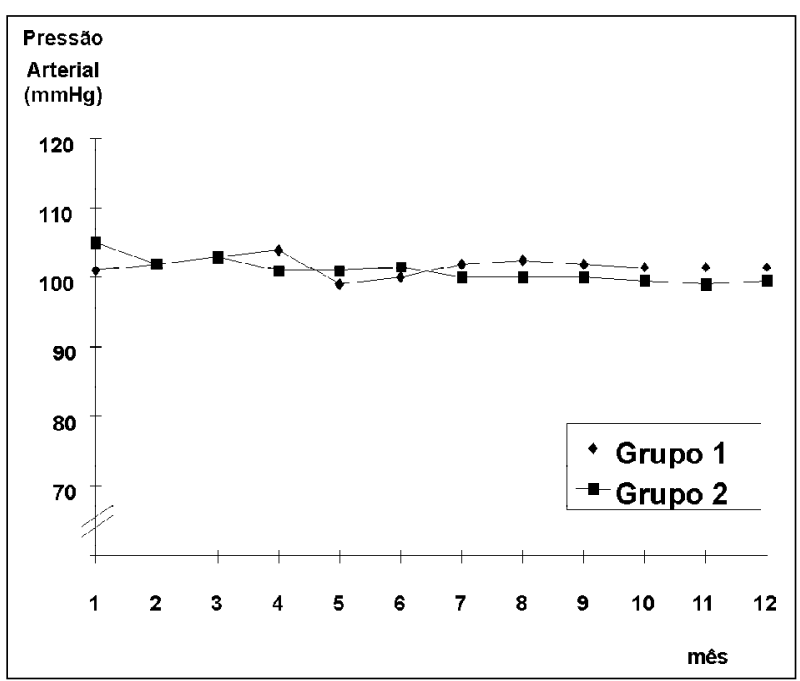

Fig. 4 - Perfil médio da pressão arterial durante repouso para o grupo 1 e grupo 2, em 12 meses de evolução. 
0,54 pelo teste de Mann-Whitney. Com base neste valor de probabilidade, não há evidência de que os grupos sejam provenientes de amostras distintas. Observa-se que o comportamento da PA em exercício não foi o mesmo que o da FC em exercício, cujos valores entre os grupos foram significativamente diferentes, como mencionado anteriormente. A tabela III mostra valores de PA em exercício, com pouca variação no grupo 1 em comparação ao grupo 2, cuja diferença inter-quartis também foi superior, bem como a amplitude geral dos dados (diferença entre os valores máximo e mínimo).

A figura 5 mostra o perfil médio da PA em exercício em ambos os grupos ao longo dos 12 meses de estudo, revelando paralelismo entre eles. Destaca-se o grupo 2 com valores médios mais elevados do que o grupo 1, cujo valor médio aos 10 meses mostrou-se ainda mais reduzido.

Com respeito à diferença entre os valores de $\mathrm{PA}$, considerando as condições de exercício e repouso, não foram encontradas razões para se considerar a existência de dois grupos distintos. Os resultados foram estatisticamente semelhantes, tanto para aqueles com até $65 \mathrm{bpm}$ em repouso, como para valores $\geq 65 \mathrm{bpm}$. Entretanto, a probabilidade de significância calculada foi de 0,0614 , próxima do limite de significância ( $\mathrm{p}=0,05)$ estabelecido neste estudo.

Com referência à correlação de Pearson, não se verificou dependência linear entre FC ePA. Entretanto, observouse nos grupos 1 e 2 grau de correlação positiva entre FC e PA em repouso exercício em nível de 62 e $71 \%$, respectivamente.

\begin{tabular}{|lllllll|}
\hline $\begin{array}{l}\text { Tabela III - Mediana, quartis (Q1 e Q3) e valores mínimo e máximo } \\
\text { de pressão arterial (mmHg) em exercício para o grupo 1 e grupo 2. } \\
\text { *N- número de pacientes }\end{array}$ \\
\hline Grupo & $\mathrm{N}$ & Mediana & $\begin{array}{c}\text { Q1 } \\
(25 \%)\end{array}$ & $\begin{array}{c}\text { Q2 } \\
(75 \%)\end{array}$ & Mínimo & Máximo \\
\hline 1 & 16 & 106 & 102 & 110 & 100 & 125 \\
2 & 31 & 110 & 100 & 115 & 90 & 150 \\
\hline
\end{tabular}

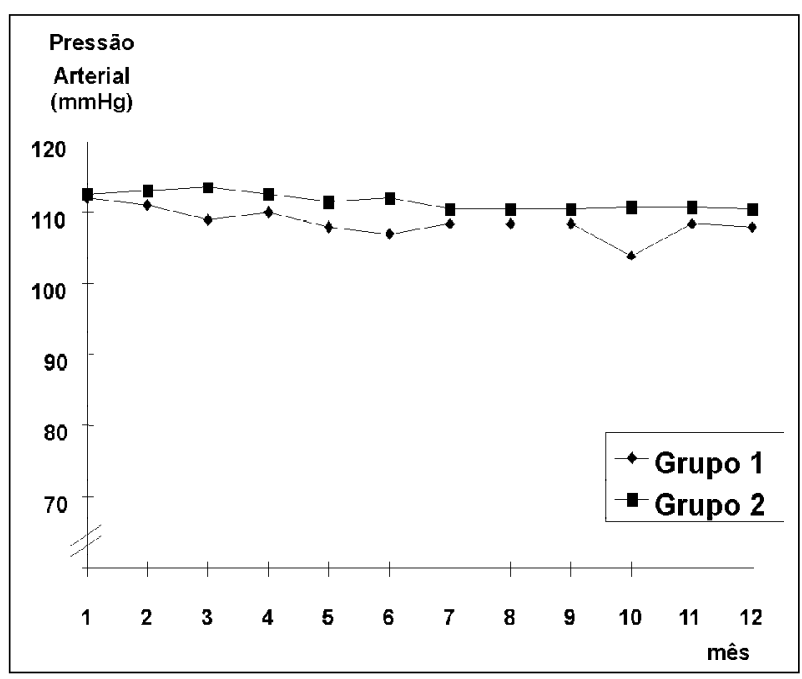

Fig. 5 - Perfil médio da pressão arterial durante exercício para o grupo 1 e grupo 2, em 12 meses de evolução.
Marcapasso com sensor de contratilidade na doença de Chagas

A figura 6 mostra a evolução dos pacientes de acordo com a classificação da NYHA, revelando melhora clínica em comparação a época do implante, com 35 (74\%) deles transferidos da CF II $(7 ; 15 \%)$, III $(16 ; 34 \%)$ ou IV $(12 ; 25 \%)$ para a CFI $(9 ; 26 \%)$, II $(23 ; 66 \%)$ ou III $(3 ; 8 \%)$. Dos 47 pacientes, $12(26 \%)$ permaneceram na mesma CF, isto é, I (4; $9 \%)$ e II $(8 ; 17 \%)$.

Quanto ao desempenho do sistema de estimulação eletrodos, o estudo comparativo entre os dois grupos de eletrodos representados por TIR-60-UP e outros foi realizado com base na diferença entre FC em exercício e em repouso de cada paciente. Com a aplicação do teste MannWhitney verificou-se diferença não significativa entre esses dois grupos de eletrodos, com base nos valores da FC pós-implante. Entretanto, a análise descritiva dos dados revelou menor variabilidade de valores referentes a diferença entre FC em exercício e em repouso, para o eletrodo do tipo TIR-60-UP, em comparação com o grupo representado por outros eletrodos.

O grau de auto-ajuste do marcapasso e sua resposta de freqüência revelaram a capacidade do sensor em aumentar a freqüência de estimulação quando solicitado. Durante as avaliações, esses pacientes tiveram vários ajustes em relação a este parâmetro, sendo que a faixa en-

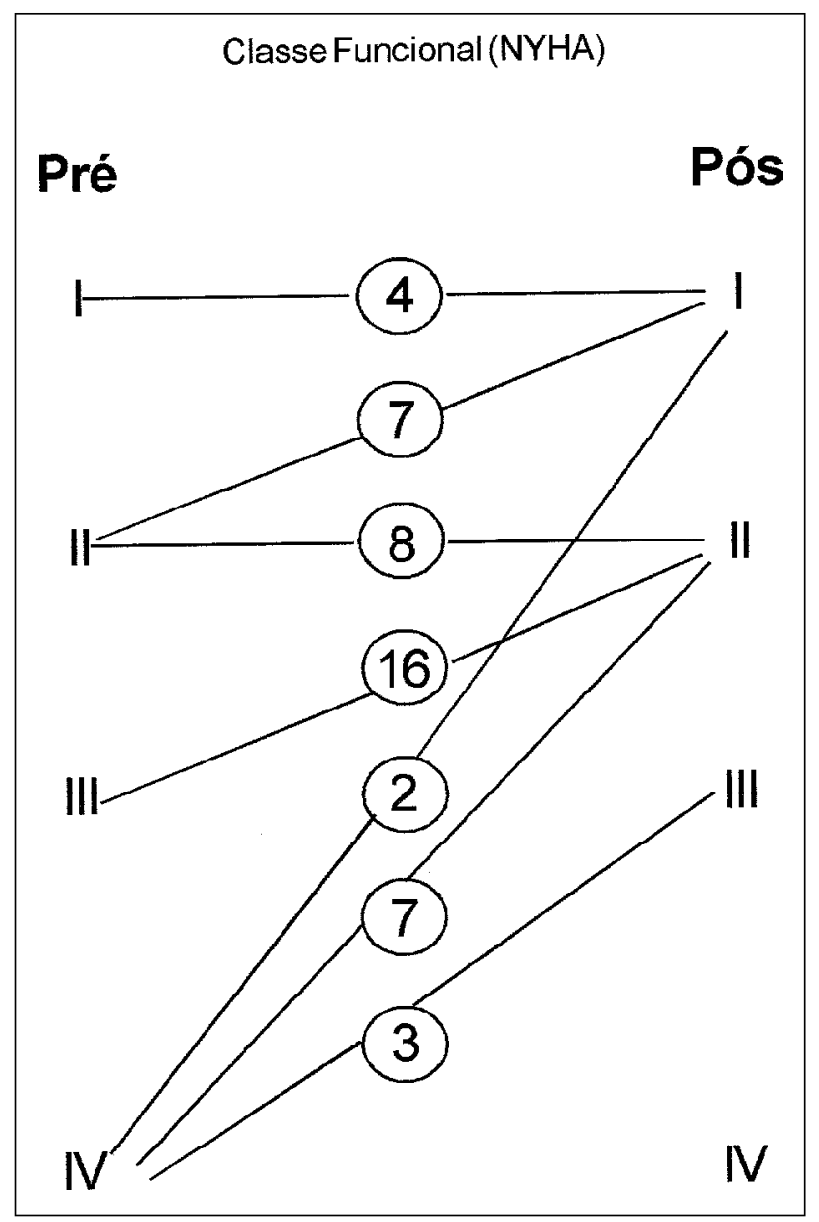

Fig. 6 - Evolução dos pacientes, de acordo com a classificação da NYHA, em 12 meses de seguimento. 
tre 0,9 e 1,1 correspondem a $68 \%$ dos ajustes ocorridos, seguida do intervalo $0,7-0,9(12 \%)$, durante o protocolo de exercício ambulatorial.

O sistema de estimulação controlado pelo SNA é sensível às variações do tônus simpático, sendo afetado não apenas por variações induzidas pelo exercício físico, mas também, por condições psicológicas, como: ansiedade, excitação e estresse mental.

A figura 7 mostra o resultado da adaptação do sistema com a gravação da FC de um paciente pela monitorização do marcapasso, paciente que foi exposto a diferentes condições de exercício em rotinas diárias. A figura 8 apresenta a monitorização de um paciente jovem durante $50 \mathrm{~min}$, com o controle apropriado da freqüência de estimulação, usando o SNA durante diferentes atividades físicas, que revelou também oscilações da frequiência de estimulação mesmo no período noturno. A figura 9 mostra a monitorização de um paciente em atividades diárias e com insônia, devido a comprometimento emocional grave.

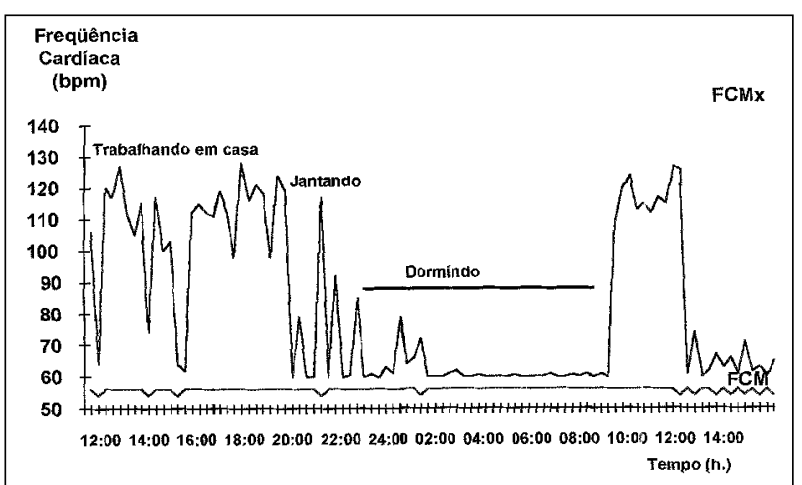

Fig. 7 - Freqüência cardíaca em batimentos por minuto (bpm), gravada por monitorização do marcapasso implantado em paciente exposto a atividades diárias rotineiras. FCM- frequiência cardíaca mínima; FCMx- freqüência cardíaca máxima.

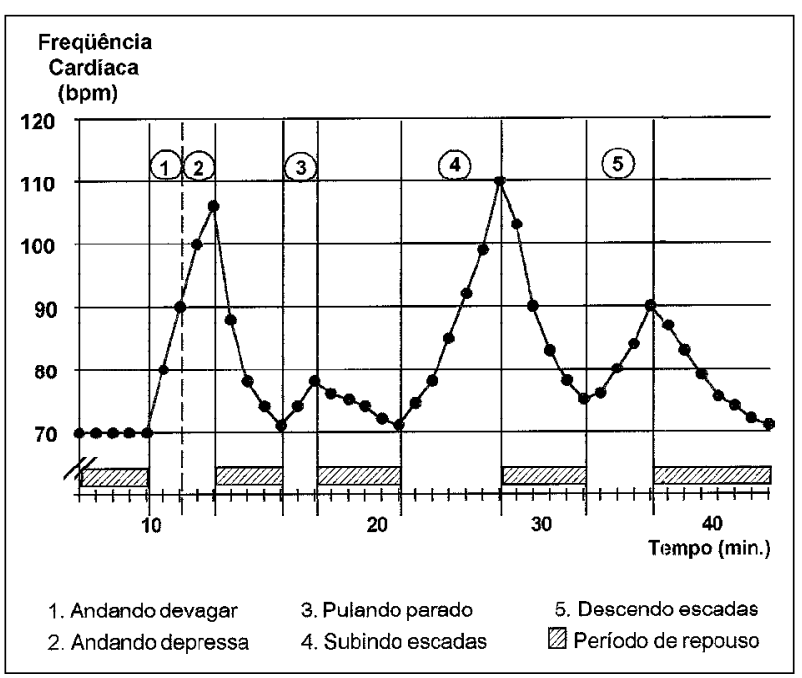

Fig. 8 - Frequiência cardíaca em batimentos por minuto (bpm), gravada por monitorização do marcapasso implantado em paciente submetido a diversas atividades, mostrando o controle adequado da freqüência de estimulação, de acordo com a atividade exercida.

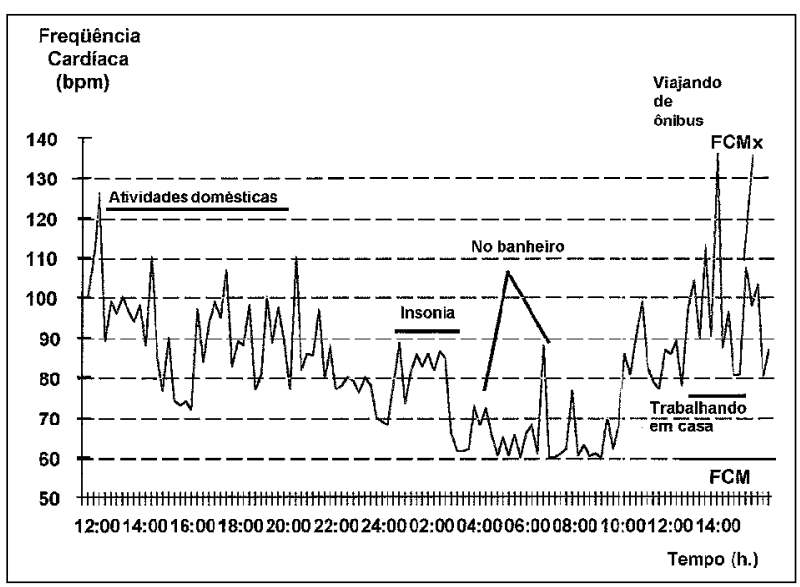

Fig. 9 - Frequiência cardíaca em batimentos por minuto (bpm), gravada por monitorização do marcapasso implantado em paciente exposto, entre outras atividades, também a situações de estresse, registrando oscilações da frequiência de estimulação. FCM- frequiência cardíaca mínima; FCMx- freqüência cardíaca máxima.

\section{Discussão}

Este estudo revelou a eficiência de um sistema de estimulação em pacientes chagásicos. A avaliação clínica durante 12 meses de seguimento mostrou a evolução satisfatória de todos os pacientes estudados, $32(68 \%)$ deles tendo atingido CF I ou II na classificação funcional de IC da NYHA. Detectou-se variação imediata da frequiência de estimulação diante de atividade física rotineira dos pacientes, avaliada por variações nas curvas obtidas com gravações de Holter de $24 \mathrm{~h}$ feitas pelo próprio marcapasso, cuja análise indicou a adaptação do sensor ao paciente e à sua atuação na modulação da freqüência de estimulação.

Fatores envolvidos nos sistemas de estimulação cardíaca - O estudo comparativo, entre os grupos com FC $>65 \mathrm{bpm}$ (grupo 1) e $\leq 65 \mathrm{bpm}$ (grupo 2) em repouso na fase pós-implante, mostrou que os pacientes do grupo 1 apresentaram em exercício freqüência de estimulação menor que os pacientes do grupo 2, evidenciando a importância da FC para o controle do sistema de estimulação por marcapasso em cada paciente. Assim, aqueles com valores elevados de FC permaneceram sob grau de estimulação reduzida, em comparação àqueles com FC baixa com necessidade de estímulo em grau superior. Muitos fatores estão envolvidos na resposta ventricular, como variações intrínsecas próprias do sistema cardiovascular de cada paciente, sua estabilidade emocional e o tipo de atividade desenvolvida. Neste caso, o sensor interfere na FC, dependendo das variações do sistema nervoso autônomo que fecha um circuito de informações para que o coração mantenha uma freqüência adequada de estimulação.

O aumento apropriado da freqüência durante exercício moderado ou acelerado pode melhorar o DC e a capacidade de tolerar o exercício, como se estivesse imitando um sincronismo atrioventricular. Esta expectativa tem sido preenchida pela melhora na capacidade de tolerar exercícios, observada durante a realização de exercício acelerado em pacientes com estimulação modo VVIR convencional. Além 
da frequiência de estimulação, a resposta de frequiência, o auto ajuste, a voltagem, a sensibilidade, o período refratário e a histerese, são parâmetros que também devem ser avaliados na fase inicial do protocolo, para a realização de uma curva de estimulação cardíaca artificial, de acordo com as necessidades momentâneas do paciente.

Por ser o coração, o órgão central de regulação do sistema cardiovascular, controla o DC em resposta aos sinais sistêmicos, principalmente aqueles vindos do sistema nervoso central. Desde que o DC é o produto da FC e do volume sistólico, o ajuste do DC é efetuado pelas médias das variações destes parâmetros. Pequenas mudanças do débito podem ser atribuídas às variações do volume sistólico, ao passo que suas maiores oscilações ocorrem por conta da FC. Para aumentar o DC, o marcapasso com resposta de freqüência deve responder aos sinais liberados pelo sistema nervoso central e por isso, deve ser avaliadoem um amplocontexto relacionado com a circulação sistêmica e sua autorregulação ${ }^{15}$.

O efeito do estresse mental pode promover mudanças profundas no funcionamento do sistema cardiovascular, particularmente na FC, provocando, às vezes, seu aumento ou sua diminuição de forma brusca, dependendo do tipo de estímulo. O mesmo efeito surge, quando do paciente, é exigida a realização de tarefas aritméticas. Esses efeitos sugerem ação simpática no sistema cardiovascular, levando com isso a maior necessidade da demanda periférica. Estes episódios foram observados em alguns dos pacientes ora estudados, com habilidade emocional importante ao chegar para reavaliação e nova programação, com elevada freqüência de estimulação ${ }^{16}$. Em alguns minutos de repouso e diálogo no sentido de tranqüilizar o paciente, detectou-se nítida e progressivamente o retorno da frequiência para níveis adequados, mostrando o real efeito do sistema nervoso autônomo e a sensibilidade desse tipo de sensor.

Processos internos e externos são capazes de alterar a resistência vascular periférica total, com a intenção de aumentar as necessidades teciduais periféricas. A diminuição desta resistência pode levar à queda da $\mathrm{PA}$, detectada pelos barorreceptores da medula oblonga. Daí saem informações para mudar o tônus simpático e vagal, com alteração da FCe do volume sistólico. Este produto leva à manutenção do DC que, com seu aumento, leva também a um aumento da PA média. Em caso de perda da função sinusal, um marcapasso com sensor fisiológico pode restaurar a resposta cronotrópica, por atender apropriadamente todos esses distúrbios ${ }^{17}$.

É reconhecido que a variação da PA tem relação direta com a frequiência de estimulação, como observado também nestes pacientes, que revela o desempenho de um paciente durante exercício físico em bicicleta ergométrica, provocando aumento da frequiência de estimulação, do ritmo sinusal e da PA média até o final do exercício (5min), com regressão progressiva destes parâmetros ao seu término.

Devido ao tipo de sensor, a característica de curva de estimulação se torna individual, para cada paciente estudado, inclusive seu comportamento emocional, para que durante a calibração sua curva de estimulação possa ser adaptada às suas necessidades diárias. Isso possibilitou analisar vários tipos de forma de estimulação, utilizando o máximo de recursos representados pelos pontos presentes nas curvas obtidas pelo Holter de $24 \mathrm{~h}$, revelando o ajuste de sua sensibilidade às necessidades momentâneas do paciente.

Funcionamento dos sistemas de estimulação cardíaca - Os marcapassos multiprogramáveis atrioventriculres possibilitam a melhora da função cardíaca, corrigindo as bradiarritmias e restaurando o sincronismo atrioventricular do coração. Entretanto, na presença de insuficiência cronotrópica ao exercício, estes benefícios se tornam limitados e, para manter este sistema regulado e DC ideal, o coração utiliza as variações do volume sistólico, que estão limitadas em aproximadamente 30 a $50 \%$ dos níveis de repouso. Conseqüentemente, um paciente com estimulação cardíaca com frequiência fixa (VOO) pode sustentar em menor grau a energia gasta durante exercício, em relação à estimulação oferecida pela adaptação da freqüência, elevando-se assim o DC. Para que isso ocorra, visando tanto adaptação cardiovascular, como melhor suporte hemodinâmico durante atividade física, os marcapassos atuais têm apresentado sensores que aumentam sua frequiência de estimulação nos momentos necessários às demandas metabólicas. Pacientes com atividade física normal e doença do nó sinusal representados por $6 \%$ neste estudo, têm também grande benefício com esses geradores, por apresentarem mudanças na regulação do aparelho cardiovascular, por meio informações que o próprio gerador recebe do organismo, devolvendo em seguida a melhor freqüência de estimulação para um determinado momento ${ }^{18}$.

O mecanismo de funcionamento desses aparelhos consiste na presença de um único microprocessador com função de contar e marcar o tempo acoplado a um programa de memória. Esses dados são armazenados em um programa que controla os parâmetros desejados, proporcionando acompanhamento adequado dos pacientes. A freqüência de estimulação é adaptada de acordo com o algoritmo, que segue uma forma linear nas variações da temperatura do sistema nervoso central. A elevação da frequiência durante um quadro emocional deve ser ajustada individualmente, já que este tipo de curva é peculiar a cada paciente, principalmente em sua fase final, que é o retorno para a freqüência de base ${ }^{19}$.

Os princípios seguidos pelo marcapasso para liberar a frequiência ideal de estimulação são regidos pelo PEP (intervalo sistólico que contém informações do controle simpático) do VD, alcançando com isto um grande significado clínico. Durante atividade física, o PEP se encurta, mantendo relação linear com o tamanho do ciclo cardíaco. A confiança deste princípio está na vantagem de se padronizar um eletrodo para a estimulação, relacionado com parâmetros ligados à circulação cardíaca. Devido à sua relação com o tônus simpático, o PEP é um parâmetro confiável para adaptar uma FC ideal durante o exercício, suficiente para manter em ordem os mecanismos de autorregulação. Esta adaptação é rápida, fisiológica e previne que as reservas miocárdicas sejam gastas desnecessariamente. Por isso, os sensores baseados nas informações do PEP permitem a manutenção estável e regulação do $\mathrm{DC}^{20}$. 
Eletrodos -Chirife foi o primeiro a sugerir na literatura, com estudo de 30 pacientes durante exercícios isotônicos e isométricos, com variação emocional, entre outros parâmetros, que um sensor que seguisse as variações do intervalo de pré-ejeção poderia manter os princípios da fisiologia cardíaca. Nossos resultados são concordantes com os seus, principalmente as curvas de estimulação obtidas em diferentes momentos da atividade física intercalados com períodos de repouso ${ }^{21}$.

Schaldach e Hutten observaram a evolução de pacientes não chagásicos com esse tipo de sensor. Estabeleceram um protocolo para avaliar as variações da FC nos vários tipos de atividade física e observaram correlação positiva entre estes dois parâmetros. Ruiter e col acompanharam 10 pacientes com esse tipo de sensor durante 18 meses com evolução satisfatória. Nossos resultados, cuja resposta de estimulação independe do tipo e tempo que o eletrodo está no VD, são concordantes com os registrados por eles. Recente revisão da literatura, apresentada por Lau e Leung, mostrou o comportamento de diferentes sensores, proporcionando aumento de $32 \%$ na capacidade de tolerância ao exercício físico ${ }^{22-24}$.

A evolução de nossos pacientes confirmou sua adaptação aos sensores empregados e melhora da sintomatologia, facilitando o desenvolvimento de atividades físicas diárias. Foram utilizados neste estudo vários tipos de eletrodos, sendo a maioria (55\%) do tipo TIR-UP-60, mas todos unipolares em VD.

Vários sinais emitidos pelo organismo são captados e apropriadamente avaliados para provocar o aumento da freqüência de estimulação por um biossensor. Geralmente, estas informações são de origem neurológica, humoral e hemodinâmica. Uma destas particularidades é o parâmetro que está relacionado com o tônus simpático, já que o estímulo eferente liberado pelo sistema nervoso central estimula a manutenção do DC, para um trabalho cooperativo com o sistema de regulação autonômico-cardiovascular. O sinal cardíaco ideal deveria ser proporcional ao tônus simpático e independente da $\mathrm{FC}^{25}$.

O posicionamento adequado do eletrodo na câmara ventricular direitaé fundamental para identificação e medida da impedância intracardíaca. Os ventrículos apresentam forma geométrica variável, dificultando a determinação exata do seu volume. A aquisição desta área da câmara ventricular é baseada na impedância intracardíaca ${ }^{26}$.

Resultados clínicos preliminares obtidos por Kruse e Ryden confirmaram que as mudanças do volume sistólico final são indicadores da mudança do DC. Ajustando a FC para suportar as mudanças no DC, o marcapasso ajusta automaticamente a reserva cardíaca, que tem mostrado uma grande vantagem no aumento do débito. A contratilidade reflete diretamente o tônus simpático do coração que, por si só, contém grande quantidade de informações associadas à regulação da circulação. Essas vantagens são proporcionais à medida da impedância intracardíaca, levada ao gerador por um eletrodo sensível às mudanças fisiopatológicas agudas ${ }^{27}$.
Perspectivas dos sistemas de estimulação cardíaca em chagásicos - A miocardiopatia chagásica crônica está classificada entre as miocardiopatias dilatadas. Quando, além do distúrbio na condução da estimulação, o paciente também apresenta falência miocárdica, seu prognóstico está comprometido mesmo com a presença de um marcapasso, como demonstrado por Lorga e $\mathrm{Col}^{28}$, que verificaram em pacientes chagásicos com cardiomegalia, sobrevida comparável à daqueles sem marcapasso. Entretanto, os marcapassos utilizados então não apresentavam a sofisticação tecnológica dos atuais, o que o presente estudo vem confirmar através da avaliação clínica satisfatória, que constatou a real adaptação desses sensores às necessidades diárias dos pacientes, em associação com as drogas atualmente disponíveis no mercado.

Embora com disfunção ventricular, os pacientes chagásicos estudados revelaram melhora em sua CF, sendo que, antes do implante, $59 \%$ localizavam-se nas CF III ou IV e, 12 meses após, 90\% nas CF I ou II, o que evidencia a presença de um sistema de alça fechada, que leva as variações periféricas à memória do sensor, independentemente da área ventricular, possibilitando o ajuste ideal da frequiência de estimulação, mesmo durante o exercício ${ }^{28}$.

A evolução satisfatória de pacientes chagásicos mantidos com marcapasso multiprogramável, assim como a melhora na sua qualidade de vida havia sido constatada por Greco e col já no final da década de 80 . Na ocasião, graças a possibilidade de mudanças da programação, evitou-se que $11,7 \%$ deles tivessem nova manipulação cirúrgica ${ }^{29}$.

Os avanços futuros na tecnologia da estimulação cardíaca têm como meta a melhora dos efeitos hemodinâmicos. Os parâmetros hemodinâmicos e funcionais são aqueles que devem ser aprimorados para que se possa entender e avaliar novos modos de estimulação. A tecnologia atual deve ser bem avaliada em alguns casos, já que o adequado intervalo AV tem auxiliado na melhora do débito em pacientes com disfunção sistólica.

No futuro, mais detalhes em relação à estimulação cardíaca com sensores e a possibilidade que o coração estimulado em certas circunstâncias pode ter um efeito terapêutico que ainda deve ser melhor explorado.

A evolução de 47 pacientes chagásicos portadores de doença no sistema de condução do coração, com implante de marcapasso cardíaco artificial dotado de eletrodo sensível a variação do sistema nervoso autônomo (SNA) permitiu, por meio de protocolo estabelecido com seguimento de 12 meses, as seguintes conclusões: 1) o marcapasso do tipo VVIR acoplado a um sensor ligado a variação do SNA restabeleceu os mecanismos fisiológicos em pacientes chagásicos com distúrbio de condução no coração; 2) esse sistema de estimulação permitiu realizar uma programação individualizada para cada um dos pacientes, proporcionandolhes uma resposta de estimulação de acordo com suas necessidades fisiológicas; 3) o eletrodo do tipo TIR-60-UP de acordo com os fatores analisados, comportou-se de modo semelhante aos demais eletrodos. 


\section{Referências}

1. Barold SS, Falkoff MD, Ong LS, Heinle RA - The third decade of cardiac pacing. BrHeart J 1981; 45: 357-64.

2. Pless P, Simonsen E, Arnsbo P, Fabricius J - Superiority of multiprogrammable to nonprogrammable VVI pacing: A comparative study with special reference to management of pacing system malfunctions. Pace 1986; 9: 739-44.

3. Sulke N, Dritsas A, Chambers J, Sowton E-Is accurate rate response programming necessary? Pace 1990a ; 13: 1031-44

4. Greco OT, Garzon SAC, Lorga AM, Ardito RV - Marcapasso de dupla câmara na cardiopatia chagásica crônica. Rev Bras Marcapasso Arritmia 1992; 5: 15-18.

5. Sant'anna JRM, Ludwig E, Ludwig, R, Lucchese FA, Schaldach M - Avaliação da fração de ejeção de ventrículo esquerdo no exercício moderado durante estimulação cardíaca ventricular e atrioventricular com resposta de freqüência. Rev Bras Marcapasso Arritmia 1992; 5: 35-40.

6. Sanchis J, Chorro FJ, Matamoros J,Monmeneu JV, Cortina J, Merino VL- Effect of site, summation and asynchronism of inputs on atrioventricular nodal conduction and refractoriness. Eur Heart J 1993; 14: 1421-6.

7. David JB, Zipes DP - Modulação Nervosa Autonômica do Ritmo Cardíaco. Parte 1 - Conceitos Básicos. Conceitos Modernos sobre Doenças Cardiovasculares 1989; 7: 1-6.

8. Rossi P - Rate-responsive pacing: Biosensor reliability and physiological sensitivity. Pace 1987; 10: 454-66.

9. Chapadeiro E, Lopes ER, Pereira EL - Denervação parassimpática e hipertrofia do miocárdio em chagásicos crônicos. Rev Inst Med Trop 1967; 9: 40-2.

10. Zipes DP, David JB - Modulação Nervosa Autonômica do Ritmo Cardíaco. Parte 2 - Mecanismos e exemplos. Conceitos Modernos sobre Doenças Cardiovasculares 1989; 7: 8-13.

11. Gallo JR L, Marin Neto JA, Manço JC, Rassi A, Amorin DS - Abnormal heart rate responses during exercise in patients with Chaga's disease. Cardiology 1975; 60: $147-62$.

12. Amorin DS, Godoy RA, Manço JC, Gallo JR L - Doença de Chagas. Enfermidade do Sistema Nervoso? Arq Bras Cardiol 1968; 21: 203-4.

13. Amorin DS, Marin-Neto JÁ - Alterações funcionais do sistema nervoso autônomo na doença de Chagas. Rev Soc Cardiol Est SP 1994; 2: 106-17.

14. Rossi AM, Bestetti RB - Hipótese unificada sobre a patogênese da cardiopatia chagásica crônica. Implicações terapêuticas. Arq Bras Cardiol 1995; 64: 255-60.
15. Benditt DG, Mianulli M, Fetter J et al - Single-chamber cardiac pacing with activity initiated chronotropic response: evaluation by cardiopulmonary exercise testing. Circulation 1987; 75: 184-91.

16. Schaldach M - Progressos na estimulação cardíaca artificial. Rev Soc Cardiol Est SP 1994; 1: 47-61.

17. Rahmoeller GA - Regulation responsive pacing- And the beat goes on. Pace 1989; $12: 1837$

18. Nordlander R, Hedman A, Pehrsson SK - Rate responsive pacing and exercise capacity. Pace 1989; 12: 749-51

19. Abrahamsen AM, Barvik S, Aarsland T, Dickstein K - Rate responsive cardiac pacing using a minute ventilation sensor. Pace 1993; 16: 1650-5.

20. Beyersdorf F, Kreuzer JTA, Happ J, Zegelman M, Satter P - Increase in cardiac output with rate-responsive pacemaker. Ann Thorac Surg 1986; 42: 201-5.

21. Chirife $\mathrm{R}$ - Physiological principles of a new method for rate responsive pacing using the pre-ejection interval. Pace 1988; 11: 1545-54.

22. Schaldach M, Hutten H - Intracardiac impedance to determine sympathetic activity in rate-responsive pacing. Pace 1992; 15: 1778-86.

23. Lau CP - The range of sensors and algorithms used in rate adaptive cardiac pacing Pace 1992; 15: 1177-1211.

24. Ruiter JH, Heemels JP, Kee D, Mechelen R - Adaptive rate pacing controlled by the right ventricular pre-ejection interval: clinical experience with a physiological pacing system. Pace 1992; 15: 886-94.

25. Furman S - Sensors in implantable cardiac devices. Pace 1991; 14: 1087-8.

26. Greco OT, Ardito RV, Lorga AM, Schaldach M - Marcapasso com biosensor que analisa a atividade do sistema nervoso autonômico através da condutância ventricular. Estudo do período de pré-ejeção. Rev Bras Marcapasso Arritmia 1992; 5: 55-61.

27. Ausubel K, Steingart RM, Shimshi M, Klementowicz P, Furman S - Maintenance of exercise stroke volume during ventricular versus atrial synchronous pacing: role of contractility. Circulation 1985; 5: 1037-43.

28. Lorga AM, Ayoub JCA, Fedozzi NM et al - BAVT chagásico com marcapasso: Estudo evolutivo de 5 anos. Arq Bras Cardiol 1976; 29(supl 1): 233.

29. Greco OT, Ardito RV, Garzon SAC et al - Acompanhamento de 991 pacientes portadores de marcapasso cardíaco artificial multiprogramável. Arq Bras Cardio 1987; 49: 327-31 